\title{
Open-Surface Digital Microfluidics
}

\author{
Jeong-Yeol Yoon*
}

Department of Agricultural and Biosystems Engineering, The University of Arizona, Tucson, Arizona 85721-0038, USA

\begin{abstract}
Open-surface digital microfluidics refers to droplet manipulations in an open surface. Different chemi$\mathrm{cal} / \mathrm{biological}$ reaction protocols can easily be demonstrated in the same device by reprogramming the order of droplet manipulations. While continuous-flow or liquid-plug microfluidics within microchannels have inherently limited programmability, there is no limit of reprogrammability in open-surface digital microfluidics. Three different mechanisms for droplet manipulations are addressed in this mini-review: electrowetting-on-dielectric, magnetofluidics and wire-guide manipulations. Brief theories of surface tension and work of adhesion are addressed. Potential applications are addressed as well as their complications.
\end{abstract}

\section{INTRODUCTION}

"Lab-on-a-chip" literally refers to a chemical or biological "lab" on a semiconductor "chip," where biological fluids flow through a miniaturized circuit (= a network of microchannels; Fig. 1 top). Therefore, microfabrication (for chip fabrication) and microfluidics (a liquid flow within microchannels) are considered as the two fundamental bases in building lab-on-a-chip. In a semiconductor chip, the circuitry can be reprogrammed to perform a different set of instructions. Similar attempts have been made in lab-on-a-chip to reprogram the reaction protocol. Microvalves and micropumps have been used for lab-on-a-chip in an attempt to simulate this reprogrammability. These "moving" components could be incorporated into lab-on-a-chip by utilizing piezoelectric components, which are very difficult to fabricate and operate [1]. Therefore, an alternative way in achieving reprogrammability has emerged: the use of discrete liquid plugs instead of a continuous liquid flow through microchannels [2-4] (Fig. 1 middle). These "plugs" are generally small in volume so that they can be considered as "droplets" or "microdrops." These droplets within microchannels have many advantages over continuous flows. Each droplet serves as a small chemical or biological reactor, where a series of different, individual reactions can be made. Very small microdrops can be created in order to confine a single cell within it (i.e. single cell analysis) [5].

However, these droplets within microchannels are not ultimate media since their movements are still restricted within microchannels. Droplets should move around in an open surface to maximize the reprogrammability of a microfluidic device (Fig. 1 bottom).

This new concept has often been coined as "digital" microfluidics. Since the manipulations of discrete liquid plugs are often referred as digital microfluidics, I will make a distinction by referring this new concept as "open-surface" digital microfluidics. Researchers at Duke University define digital microfluidics as follows: "Digital microfluidics is an

\footnotetext{
*Address correspondence to this author at the Department of Agricultural and Biosystems Engineering, The University of Arizona, Tucson, Arizona 85721-0038, USA; E-mail: jyyoon@email.arizona.edu
}

alternative technology for lab-on-a-chip systems based upon micromanipulation of discrete droplets. Microfluidic processing is performed on unit-sized packets of fluid which are transported, stored, mixed, reacted, or analyzed in a discrete manner using a standard set of basic instructions [6]."


Fig. (1). Top: Continous liquid flows through microchannels. Sample (bottom inlet) is gradually mixed with diluent (top inlet) to achieve serial dilutions. No reprogrammability. Middle: Discrete liquid plugs or droplets within microchannels. Limited reprogrammability. Bottom: Droplets on an open surface. Merging, splitting and mixing (by rotation) are shown. Maximum reprogrammability.

Examples of complex chemical/biological procedures include: (1) serial dilution, (2) potentiometric or conductometric titration, (3) chemical synthesis, (4) enzyme-linked immunosorbent assay (ELISA), (5) polymerase chain reaction (PCR) and (6) single cell analysis. Like a set of instructions for a microprocessor, these procedures can be built up step-by-step in reprogrammable lab-on-a-chip. In a way, digital microfluidics is analogous to traditional bench-top protocols while continuous-flow microfluidics is analogous 
to flow-injection analyses (in much smaller volumes and with higher level of automation).

\section{BASIC MANIPULATIONS}

\section{Basic Droplet Manipulations: Transport, Merging, Split- ting and Creation}

In order to fully perform chemical/biolgical reactions in digital microfluidics, the following droplet manipulations should be demonstrated [7]. Primarily, we should be able to move droplets (transport). Secondly, we should be able to merge droplets to simulate addition of liquids and split them to simulate taking aliquots (merging and splitting). Finally, we should be able to create droplets from either inlets or small reservoirs (creation) (Fig. 2). This creation can be made manually by using micro-pipettes when the volume of droplets is larger than $1 \mu \mathrm{L}$. Nanoliter drops should be created automatically within digital microfluidics.

\section{Mixing and Heating}

Since we do not have a magnetic stirrer, sonicator, or vortex mixer, we should be able to have certain mechanism to achieve mixing for a merged droplet. Molecules may spontaneously diffuse to the other side of a merged droplet towards complete mixing; however, this diffusional mixing is inappropriate for biomolecules (their very high molecular weights make their diffusivity very low). Turbulent mixing seems to be the best solution in achieving mixing, and one such attempt was made possible by rotating a droplet clockwise then by counterclockwise [8] (Fig. 2).

Some chemical/biological reactions require heating for incubating cells at certain optimum temperature, denaturation of nucleic acids, and most notably, PCR. This heating may be achieved by placing a metal electrode in a certain area of an open surface and applying electricity to it. The passage of an electric current through a conductor releases heat, which is known as Joule heating (Fig. 2).

Fig. (2) also illustrates optical detection using a backscattering/reflection probe, which will be discussed later in this review.



Fig. (2). Transport, merging, mixing, heating and optical detection of droplets.

Once these basic manipulations are made possible, one can control the movement of droplets using a pre-defined computer program through a digital interface. Alternatively, the movements can be controlled in real time from an external computer (direct commands). Fig. (3) illustrates one of such possible confugurations, where a droplet follows the stylus movement in a handheld computer through wireless connection.

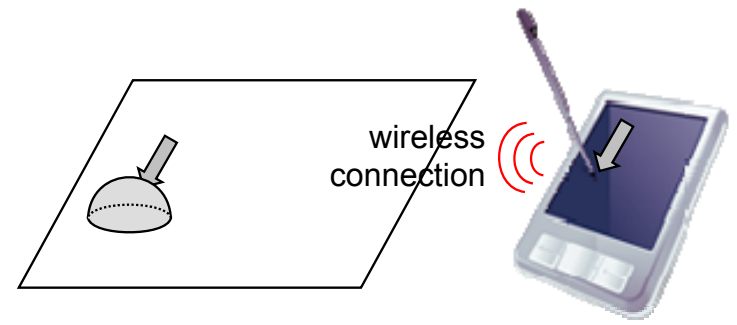

droplet follows the stylus movement in PDA

Fig. (3). Droplet manipulation using wireless connection and a handheld computer (PDA; personal digital assistant).

\section{ACTUATION METHODS}

\section{Contact Angle and Surface Tension}

Whether we are able to manipulate a droplet or not depends primarily on the work of adhesion $W_{a}$, defined by Young-Dupré equation $W_{a}=\gamma_{L}(1+\cos \theta)$, where $\gamma_{L}$ is liquid surface tension and $\theta$ is contact angle, as defined in Fig. (4) [9]. Young-Dupré equation tells us how much work should be provided to overcome $W_{a}$ of a droplet to a surface, which is primarily a function of $\theta\left(\gamma_{L}\right.$ is constant if the liquid is mostly water).

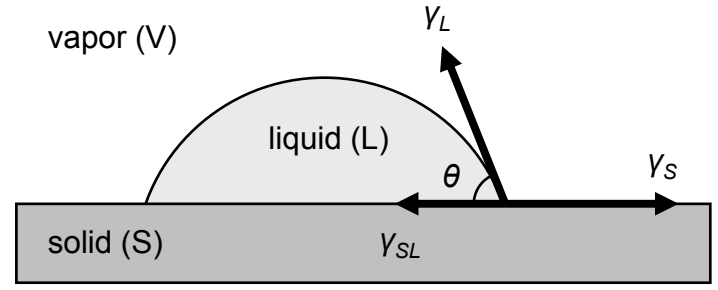

Fig. (4). Contact angle and surface tensions.

\section{Electrowetting-On-Dielectric (EWOD)}

EWOD for digital microfluidics has been extensively studied by a group of scientists at University of California, Los Angeles (UCLA; most notably Chang-Jin Kim) and Duke University (most notably Richard B. Fair). In EWOD, droplets move on a chip surface through electrical modification of the surface hydrophobicity [10, 11]. A Si wafer is etched into patterned 2-D grids, and two dielectric layers (silicon dioxide and Teflon) are added to insulate electricity thus preventing the electrolysis of water. Applying an electrical voltage to one of the electrodes causes the surface tension between solid and liquid $\gamma_{S L}$ to decrease, thus lowering $\theta$ [12]. Theoretically, the contact angle can be decreased as low as $0^{\circ}$ as long as high voltage is applied. Practically, however, the contact angle can only be decreased down to $80^{\circ}$ from the initial $115^{\circ}$ (that of Teflon), due to contact angle saturation and dielectric breakdown [12]. This $35^{\circ}$ decrease still sufficiently enables the droplet to be attracted to the electricity-charged electrode (Fig. 5).

Since the voltage is applied to one of the electrodes, a ground electrode becomes necessary. This can be achieved in 
two different ways, namely, by a network of guide wires or by a conductor cover slide, typically indium tin oxide (ITO) $[2,13]$, as shown in Fig. (6).



electrowetting-on-dielectric (EWOD) superparamagnetic particles

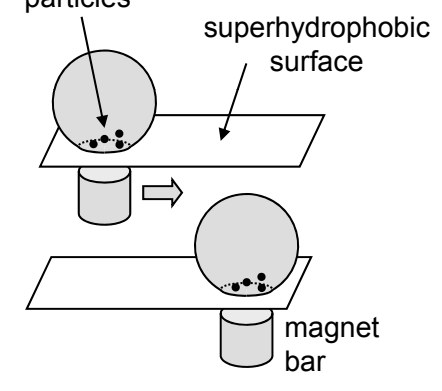

magnetofluidics
Fig. (5). Electrowetting-on-dielectric (EWOD) and magnetofluidics.

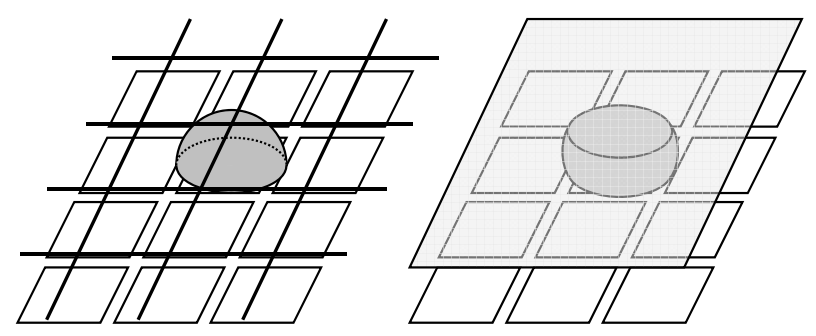

Fig. (6). Ground electrodes for EWOD. Left: a network of guide wires; Right: a conductor cover slide.

Moving, merging, splitting, creation, and mixing of droplets have all been demonstrated [7,8]. Contact angle saturation and the choice of dielectric layers have been studied [12, 14]. The use of biomolecules within droplets was also investigated [15]. The use of nonaqueous solvents was attempted [16]. For biological assays with EWOD, refer to the Applications section.

\section{Magnetofluidics}

Digital magnetofluidics has demonstrated by a research team from Universidad Nacional de Educación a Distancia (UNED; most notably Miguel A. Rubio) and Arizona State University (ASU; most notably Antonio A. Garcia). In magnetofluidics, droplets move on a chip surface through the use of magnetic fields. This is achieved by adding superparamagnetic particles to a droplet placed on a superhydrophobic surface with a moving magnet bar underneath [17] (Fig. 4). Since the magnetic force between magnetic particles and a magnet bar is very weak, a superhydrophobic surface (whose $\theta$ is close to $150^{\circ}$ or higher) is needed in order to minimize both the contact area and the work of adhesion for a droplet (the latter is often defined as "frictional force"). Recently, however, simple perfluorinated surface $\left(\theta=115^{\circ}\right)$ has also successfully been used with the help of stronger magnetic beads and emersion of an aqueous droplet into silicone or mineral oil [18]. Due to its simplicity, many different biological assays have been demonstrated with this setup, in spite of its relatively short history. For biological assays with magnetofluidics, refer to the Applications section.

\section{Wire-Guide Manipulations}

In EWOD, fabrication of a robust dielectric layer is required to prevent the electrolysis of water and subsequent device failure [12]. In magnetofluidics, paramagnetic particles should be designed not to interfere with biological reactions, a capability that has not been confirmed yet. In both cases, external electric or magnetic fields may affect the behavior of biomolecules, and, consequently, bioanalysis and biorecognition.

A simpler method for manipulating droplets on a flat surface is needed to minimize the complications described above. A clean, metal wire $\left(\theta<10^{\circ}\right)$ may be inserted into a droplet to guide its movement on a surface. However, the $W_{a}$ of a droplet to a metal wire is simply too small to overcome the $W_{a}$ to a flat surface due to the small contact area between the droplet and wire. Since $W_{a}=\gamma_{L}(1+\cos \theta)$, a very large $\theta$ may make this movement possible. We have recently demonstrated such droplet manipulations on a superhydrophobic surface [19].

A superhydrophobic surface was used in both magnetofluidics and wire-guide manipulations, that are made from a nanocoatings of a fluoropolymer on standard glass microscope slides and are currently available commercially. These nanocoatings create surface air pockets and put a droplet in metastable Fakir state. These air pockets are considered to be completely non-polar (i.e. hydrophobic), increasing $\theta$ as high as $150^{\circ}[20]$.

\section{Surfance Tension and the Work of Adhesion}

From $W_{a}=\gamma_{L}(1+\cos \theta)$, the adhesive energy is a sole function of $\theta$ for the same type of liquid and its contact area. The higher $\theta$ is, the lower $W_{a}$ becomes. In case of EWOD, $\theta$ changes from $115^{\circ}$ (that of Teflon) to $80^{\circ}$ (with voltage) [12, 15], $\gamma_{L}=73 \mathrm{~mJ} \mathrm{~m}^{-2}$ for water, and typical area of an electrode is $1 \mathrm{~mm}^{2}$ with typical droplet volume of $0.4 \mu \mathrm{L}$. $W_{a}$ can accordingly be calculated as $42 \mathrm{~nJ}$ with no voltage applied and $86 \mathrm{~nJ}$ with voltage applied (Table 1). The energy with voltage applied is approximately twice that with no voltage applied. Therefore, a proper contact area to the actuated electrode can make the droplet movement possible. This situation is schematically illustrated in Fig. (7 top).

In magnetofluidics, it is difficult to calculate the exact magnetic energy between paramagnetic particles and a magnetic bar as shown in Fig. (7 bottom left). Since magnetofluidics has not been successful with conventional plastic surfaces such as Teflon $\left(\theta=115^{\circ}\right)$, we can assume such magnetic energy is generally too weak to overcome the work of adhesion for many conventional surfaces.

In wire-guide droplet manipulations, the contact area of a droplet to a metal wire is much smaller than that to a surface, as shown in Fig. (7 bottom right). Therefore, a large contact angle difference between a metal wire and a surface is required. We have recently demonstrated such manipulations of $10 \mu \mathrm{L}$ drops using a clean metal wire $\left(\theta \approx 10^{\circ}, 0.5 \mathrm{~mm}\right.$ $\mathrm{OD}$, inserted into a droplet by $2 \mathrm{~mm}$, contact area $=0.39$ $\left.\mathrm{mm}^{2}\right)$ and a superhydrophobic surface $\left(\theta=145^{\circ}\right.$, contact area $=2.0 \mathrm{~mm}^{2}$ ). Note that the contact area between a droplet and a superhydrophobic surface is considerably small, compared to that on a polystyrene (PS) surface $\left(\theta=90^{\circ}\right.$, contact area $=$ 
$\left.10.5 \mathrm{~mm}^{2}\right)$ and a Teflon surface $\left(\theta=115^{\circ}\right.$, contact area $=6.9$ $\mathrm{mm}^{2}$ ) for the same $10 \mu \mathrm{L}$ droplet. $W_{a}$ was calculated as $57 \mathrm{~nJ}$ to a metal wire and $26 \mathrm{~nJ}$ to a superhydrophobic surface, enabling for a droplet to move with a metal wire. Such demonstration was not possible with other plastic surfaces due to their large $W_{a}, 290 \mathrm{~nJ}$ (Teflon) and $750 \mathrm{~nJ}$ (PS), both are larger than that to a metal wire (57 $\mathrm{nJ})$.

Table 1. Work of Adhesion $\left[W_{a}=\gamma_{L}(1+\cos \theta)\right]$ for EWOD and Wire-Guide Manipulations. $\gamma_{L}=73 \mathrm{~mJ} \mathrm{~m}^{-2}$, Droplet Volume $=0.4 \mu \mathrm{L}$ for EWOD and $10 \mu \mathrm{L}$ for Wire-Guide

\begin{tabular}{|c|c|c|c|}
\hline EWOD & $\boldsymbol{\theta}$ & Cont. area & $\boldsymbol{W}_{\boldsymbol{a}}$ \\
\hline \hline No voltage & $115^{\circ}$ & $1.0 \mathrm{~mm}^{2}$ & $42 \mathrm{~nJ}$ \\
\hline Voltage applied & $80^{\circ}$ & $1.0 \mathrm{~mm}^{2}$ & $86 \mathrm{~nJ}$ \\
\hline Wire-Guide & $\boldsymbol{\theta}$ & Cont. area & $\boldsymbol{W}_{\boldsymbol{a}}$ \\
\hline \hline Metal wire & $10^{\circ}$ & $0.39 \mathrm{~mm}^{2}$ & $57 \mathrm{~nJ}$ \\
\hline Superhydrophobic & $145^{\circ}$ & $2.0 \mathrm{~mm}^{2}$ & $26 \mathrm{~nJ}$ \\
\hline Teflon & $115^{\circ}$ & $6.9 \mathrm{~mm}^{2}$ & $290 \mathrm{~nJ}$ \\
\hline PS & $90^{\circ}$ & $10.5 \mathrm{~mm}^{2}$ & $750 \mathrm{~nJ}$ \\
\hline
\end{tabular}
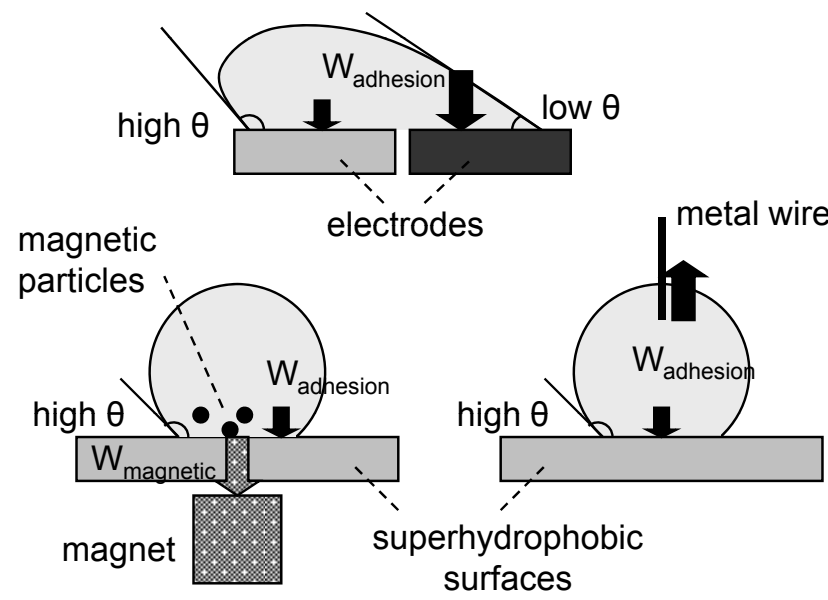

Fig. (7). $W_{a}$ for EWOD (top), magnetofluidics (bottom left) and wire-guide manipulations (bottom right).

\section{Detection: Electrochemical Versus Optical}

Regardless of whether reactions are chemical or biologi$\mathrm{cal}$, all end products need to be quantified to generate tangible results. This is a biosensor topic, so we can think of all possible biosensing modalities: electrochemical, optical, piezoelectric and thermal. Of course the first two (electrochemical and optical) are the most popular, since the other two (piezoelectric and thermal) are too complex to be implemented in digital microfluidics. Electrochemical sensing involves with oxidation/reduction cycles of a substrate (e.g. hydrogen peroxide; $\mathrm{H}_{2} \mathrm{O}_{2}$ ) with an enzyme (e.g. horseradish peroxidase; HRP). This cycle consumes or produces electrons that can be detected with amperometry (electric current) or potentiometry (electric voltage). Therefore it becomes necessary to have an acting electrode with a reference electrode. This is relatively easy to accomplish in EWOD, since acting and ground electrodes already exist. In case of magnetofluidics and wire-guide manipulations, a droplet should make a contact to both electrodes to detect electric current, voltage or resistance. This two-electrode contact is generally difficult to achieve, since the contact area of a droplet to a surface is relatively small for a superhydrophobic surface $\left(\theta \approx 150^{\circ}\right)$. Additionally, the use of a cover slide similar to that in EWOD may not be a good solution, since the work of adhesion will be increased twice by additional contact of a droplet to a cover slide.

Optical detection is a good alternative for the droplet manipulations on a superhydrophobic surface. Molecules labelled with fluorescent dyes or quantum dots can be detected with a photodiode (PD), Avalanche photodiode (APD), photomultiplier tube (PMT), charge-coupled device (CCD), or complementary metal-oxide-semiconductor (CMOS). Our group has also demonstrated the use of optical fibers and a miniature spectrometer (essentially a one-dimensional array of $\mathrm{CCD}$ ) for quantifying latex immunoagglutination assays in microchannel devices [21-23]. We have recently demonstrated the use of a backscattering/reflection probe for wireguide droplet manipulations, which is essentially a core-shell bundle of optical fibers attached to a miniature spectrometer [19]. Light is delivered through the core fiber towards a droplet and any scattered, reflected or emitted light is collected to the shell-side fiber bundles. The distance and location of a probe from a droplet should be optimized such that the maximum optical signal is attained. This optimization may be achieved through computer-controlled positioning stages with stepping motors (Fig. 8).
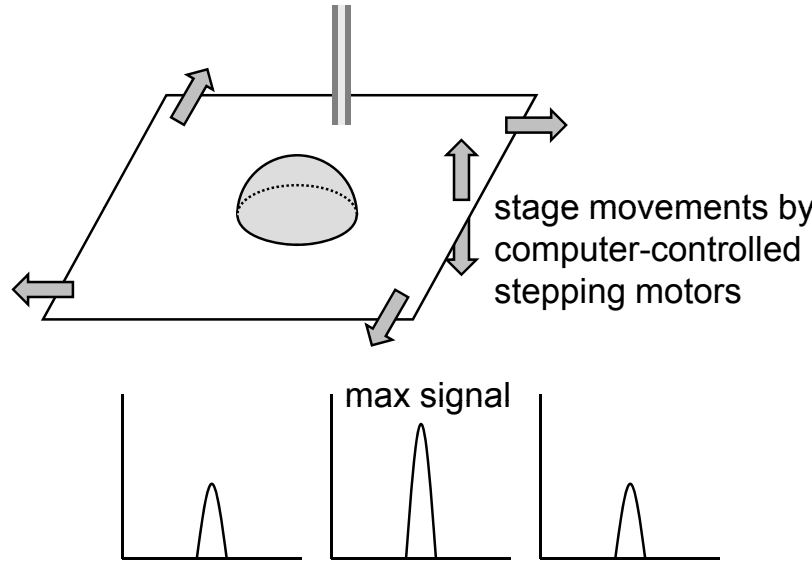

spectra as calibrating droplet location over $\mathrm{x}$-axis (repeated over $y$ - and z-axis)

Fig. (8). Maximizing the optical signal from a droplet using a backscattering/reflection probe and computer-controlled positioning stages.

In EWOD, a transparent cover slide is preferred over a network of guide wires (refer to Fig. 6) for optical detection. A backscattering probe may be located on top of a cover slide. If the bottom substrate is also transparent, incident light may be delievered to the bottom, while detection is made at the top. ITO is probably the most popular conductor material that is optically transparent [13].

\section{APPLICATIONS}

\section{Titrations}

Titration is one of the most popular chemical analyses, and modern titrations are performed using a potentiometer 
(including a $\mathrm{pH}$ meter) or a conductometer. Titrant should continously be added to an analyte, which makes its demonstration in microfluidic devices rare, especially in the continuous-flow condition. Serial dilutions of an analyte with a titrant is required, which may be possible in digital microfluidics. We propose one possible protocol in digital microfluidics for potentiometric/conductometric titrations in Fig. (9). Two drops are created from analyte and titrant reservoirs, followed by splitting into halves. One each of the split drops are merged, mixed, and split again. One of the split-merged drop is sent to the detection site to record its $\mathrm{pH}$ or conductivity, where the other is merged with an analyte or a titrant to make a different dilution.
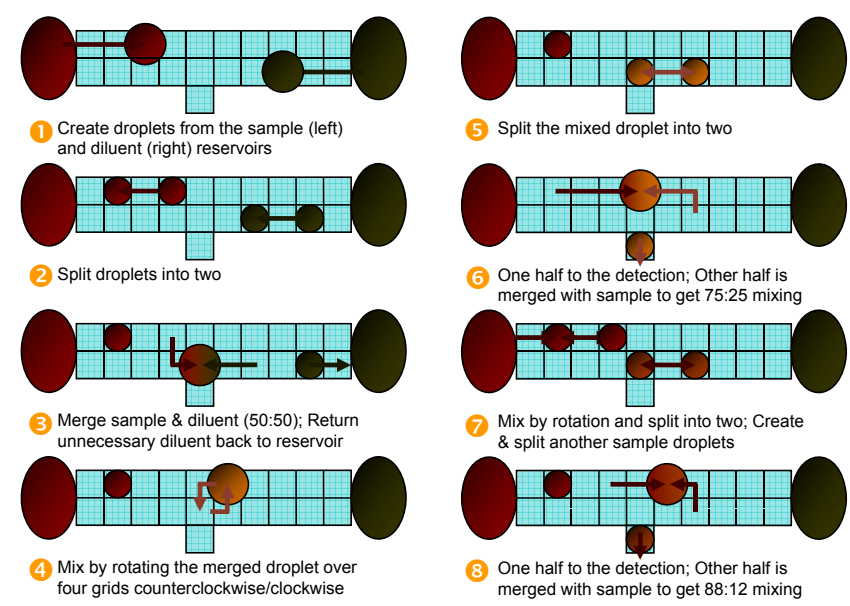
(5) Split the mixed droplet into two

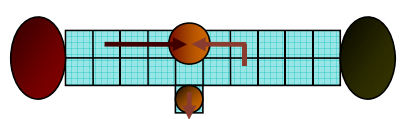
6) One half to the detection; Other half is


(7) Mix by rotation and split into two; Create \& split another sample droplets

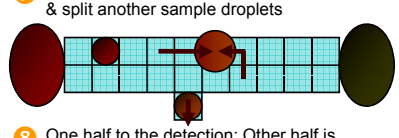

8 One half to the detection; Other half is
merged with sample to get $88: 12$ mixing

Fig. (9). Proposed protocol for a potentiometric or conductometric titration in digital microfluidics.

\section{Glucose Sensing}

Glucose sensing is probably the most-studied topic in biosensors. Consequently there have already been a few attempts of glucose sensing in digital microfluidics in both EWOD [24] and magnetofluidics [25]. Glucose is quantified by detecting its oxidation byproduct, hydrogen peroxide. Both electrochemical and optical detections are possible in quantifying this hydrogen peroxide. Electrochemincal detection was demonstrated in magnetofluidics by inserting two adjacent electrodes into a droplet, for 2-20 mM glucose [25]. Optical detection was demonstrated in EWOD using a light emitting diode (LED) and a photodiode (PD), for 20-100 mg $\mathrm{dL}^{-1}$ glucose [24]. Such detections are very likely to be demonstrated in wire-guide manipulations, although no such attempts have been made yet.

\section{Immunoassays}

The immunoassay utilizes the binding of antigens to antibodies towards detection of a given molecule or organism, and is one of the most popular methods in clinical diagnostics and environmental monitoring. A wide variety of immunoassay protocols are currently available for laboratorybased analyses. These include enzyme linked immunosorbent assay (ELISA, the most popular) and particle agglutination. Many commercial kits for immunoassays, for example ELISA, require the antibody (or antigen) to be immobilized on a fixed surface so that the unbound molecules can easily

be washed away. Although a little rare, ELISA was attempted in magnetofluidics platform, for detecting $0.6 \mathrm{ng}$ $\mathrm{mL}^{-1}$ (or $6 \mathrm{pg}$ in a $10 \mu \mathrm{L}$ droplet) antibodies conjugated with HRP [26].
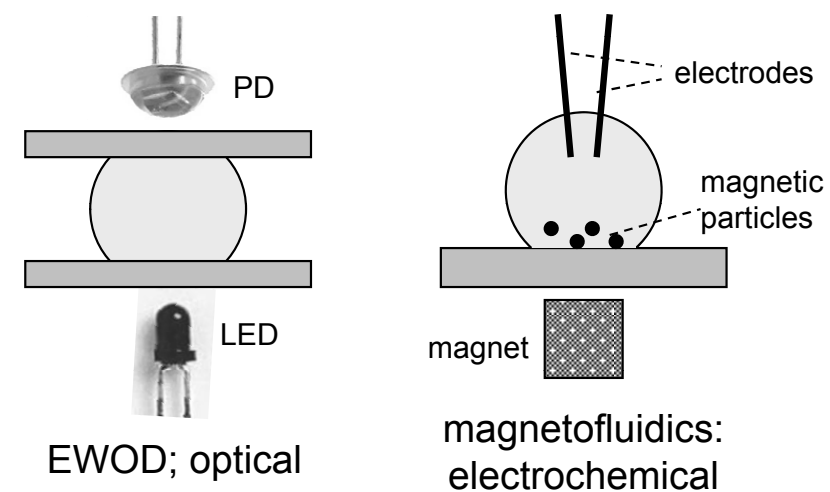

magnetofluidics: electrochemical

Fig. (10). Optical detection of glucose in EWOD (left) and electrochemical detection of glucose in magnetofluidics (right).

There have been other attempts in utilizing micro- or nanoparticles in assaying biomolecules with no fixed-surface immobilization. Latex immunoagglutination assays, also known as latex agglutination tests, is such an attempt. In a latex immunoagglutination assay, antigen-antibody binding causes microparticles (also known as latex beads or microspheres) to be agglutinated to form a doublet or a larger cluster. This agglutination can be visualized or quantified through light scattering detection. Typical diameters of microparticles range from $100 \mathrm{~nm}$ to $10 \mu \mathrm{m}$ [27]. Latex immunoagglutination assays have been demonstrated in wireguide manipulations for antibody (detection limit $=50 \mathrm{pg}$ $\mathrm{mL}^{-1}$ or $0.5 \mathrm{pg}$ for a $10 \mu \mathrm{L}$ droplet), virus (bovine viral diarrhea virus; BVDV; detection limit $=2.5 \mathrm{TCID}_{50} \mathrm{~mL}^{-1}$ or $0.025 \mathrm{TCID}_{50}$ for a $10 \mu \mathrm{L}$ droplet) and bacterium (Escherichia coli; detection limit $=85 \mathrm{CFU} \mathrm{mL}^{-1}$ or $<1 \mathrm{CFU}$ for a $10 \mu \mathrm{L}$ droplet) by merging two droplets each containing antibody-conjugated microparticles and target molecules (Fig. 11 top) [19].

Although not an immunoassay, mass spectrometry of protein has also been demonstrated in an EWOD platform [28].

\section{Polymerase Chain Reaction}

Although antibody-based immunoassays are simple yet very sensitive, their specificity has always been questioned. Depending on many factors, especially antibody quality, an immunoassay result may become false-positive. Sequencing nucleic acids (DNA or RNA) of a target would be an ultimate alternative in improving specificity, at the cost of increased difficulty and inferior sensitivity. Polymerase chain reaction (PCR) has probably eliminated these complications, by easily amplifying the amount of target nucleic acid sequence. Conseuqenly, a few papers have been published for PCR demonstrations in both EWOD [29] and magnetofluidics [30,31] (Fig. 11 bottom). For both cases, aqueous droplets were immersed in silicone or mineral oil to prevent possible evaporation upon heating. A thermocycler chamber was used for the most of these demonstrations, similar to the PCR demonstrations in liquig-plug type microfluidics [32]. Pipper et al. [31], however, attempted this thremocycling by 
moving a droplet over a temperature gradient, rather than changing the temperature of a single location (i.e. chamber).
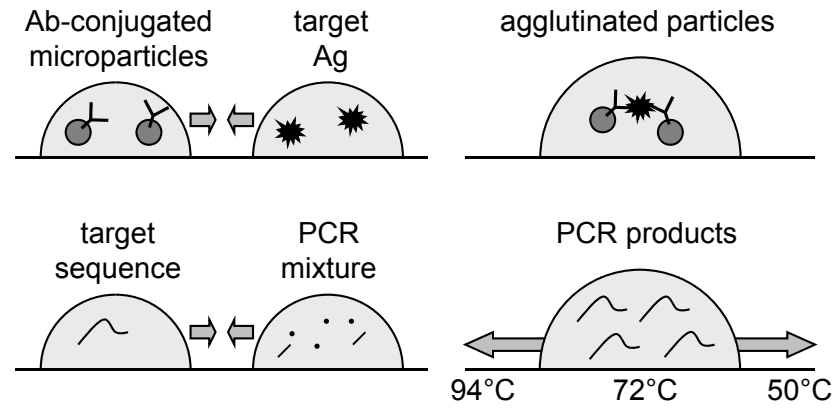

Fig. (11). Demonstration of latex immunoagglutination assay (top) and PCR (bottom) in digital microfluidics.

\section{Single Cell Analysis}

There have been a few attempts to confine a single cell in a liquid drop within a microchannel, although not on an open surface. Droplets are separated by oil, providing miniature, isolated reaction compartments. Creation of nano-, pico-, or even femtoliter drops makes this miniature compartment similar to natural environment, and makes the concentration of signaling proteins relatively high. E. coli were identified with fluorescence [33], and photolysis of mouse mast cells and B lymphocytes were attempted [34] in liquid-plug microfluidics.

Single cell analysis has not yet been demonstrated in open-surface digital microfluidics (EWOD, magnetofluidics or wire-guide manipulations). Obviously, the enhanced reprogrammability is an advantage for analysing single cells on open-surface digital microfluidics. Since very small volume is required, droplets should be immersed in oil to prevent possible evaporation.

\section{COMPLICATIONS}

\section{Evaporation}

Since droplets have a small volume, they can evaporate relatively quickly upon exposure to air (especially for nanoor picoliter drops required for single cell analysis). Evaporation is also a serious issue in PCR demonstration due to the need for high temperature heating of the droplet. To prevent this evaporation, droplets have been immersed in silicone or mineral oil [29,30,31]; however, oil immersion is not always beneficial due to the following complications: (1) fast droplet transport is not possible (required for turbulent mixing) and (2) proximity optical detection is difficult due to the possible interference by oil.

\section{Biofouling and Contact Line Pinning}

Over time, many biomolecules that have hydrophobic surfaces irreversibly adsorb to not only the air-liquid interface (oil-liquid interface in case of oil immersion) but also to the liquid-solid interface, through hydrophobic interactions. We have to recall that the droplets in digital microfluidics are always exposed to strongly hydrophobic surfaces, e.g. air, oil, Teflon, perfluorinated or superhydrophobic surfaces. This "passive" adsorption (biofouling) makes $\theta$ lower by at least $15-20^{\circ}$ [15]. The more serious problem is, however, that biofouling is concentrated at the three-phase borderline (contact line) which causes the contact line to become pinned. Since the contact line should be able to move around freely for droplet manipulations, this contact line pinning eventually undermines the overall performance of droplet manipulations.

Making the surface hydrophilic, i.e. modifying the surface with polyethylene glycol (PEG), is not an option for digital microfluidics, as the surfaces should be made hydrophobic for EWOD, magnetofluidics and wire-guide manipulations. Two approaches have suggested by Yoon and Garrell [15]: (1) Minimize the contact time for passive adsorption, since passive biofouling is relatively slow (occurs over several minutes). (2) Cause the protein molecules to be repelled from the bottom surface through adjustment of the medium $\mathrm{pH}$ and the surface charge of the bottom substrate. The net charge of a protein molecule changes from positive to negative as the medium $\mathrm{pH}$ passes through the isoelectric point $(\mathrm{pI})$ of a protein.

\section{Electrolysis}

Electrolysis is solely an EWOD problem. Although there is a dielectric layer that can insulate an electric current, this layer can be broken down (dielectric breakdown) upon repeated exposure to electric voltage. Once this failure happens, electric current flows from the acting to the ground electrodes, and water electrolysis follows, generating oxygen and hydrogen gases. Since electrolysis of pure water is known to be very slow, electrolysis is not a big problem in manipulating pure water drops in EWOD. However, addition of buffer or protein molecules to the water dramatically increases the extent of electrolysis in EWOD.

The only solution to prevent electrolysis is to make the dielectric layer as robust as possible. Spin coating of thick Teflon has proven to be the worst in preventing dielectric breakdown. Chemical vapor deposition (CVD) of silicon dioxide together with Teflon coating was demonstrated to be a little better. The best solution was oxidizing the top layer of silicon wafer into silicon dioxide (thermal oxidation) together with hydrophobic Teflon coating [12].

\section{Effects of Electrical and Magnetic Fields}

Since electric voltage is being applied to each electrode in EWOD, all biomolecules within a droplet are exposed to the electric field. Depending on the medium $\mathrm{pH}$, the net charge of protein molecules may be varied from positive to negative, depending on the isoelectric point of the proteins. If these electrostatic interactions are not properly controlled, protein molecules may adsorb to the bottom surface and especially to the three-phase borderline, leading to biofouling and contact line pinning and eventually no movement of droplets.

In magnetofluidics, biomolecules are exposed to magnetic field. Since many biomolecules are neither ferro- nor paramagentic, the possibility of biofouling and contact line pinning due to magnetic force is very low. The only concern is the possible interaction between biomolecules and paramagnetic particles, where the particles are attracted to and move around the bottom surface. To the best of my knowledge, no systematic studies have been reported in addressing these issues. 
In wire-guide manipulations, there are obviously no effects of electric/magnetic fields.

\section{CONCLUSION}

There are many advantages in manipulating droplets on an open surface, in comparison to a microchannel. A wide range of established chemical or biological protocols can be demonstrated without any restrictions on an open-surface digital microfluidic platform. This is still a very young discipline; EWOD manipulation is less than 7 years old; magnetofluidics less than 2 years; and wire-guide manipulation has just emerged. A new kind of manipulation may emerge soon as well. It is likely that scientists and engineers will be attracted to the fact that many established chemical or biological protocols are still waiting to be tested in these new platforms.

\section{ACKNOWLEDGMENT}

The author is grateful for Professor Robin L. Garrell at University of California, Los Angeles and Professor Antonio A. Garcia at Arizona State University for helpful discussions. The funding for this work was provided by National Veterinary Research and Quarantine Service (NVRQS), Republic of Korea, Award No. C-AD14-2006-11-1.

\section{REFERENCES}

[1] Madou MJ. Fundamentals of microfabrication, 2nd edn., CRC, Boca Raton, FL, 2002.

[2] Berthier J, Silberzan P. Microfluidics for Biotechnology, Artech House, Norwood, NJ, 2006.

[3] Belder D. Microfluidics with droplets. Angew Chem Int Ed 2005; 44(23): 3521-3522.

[4] Joanicot M, Ajdari A. Droplet control for microfluidics. Science 2005; 309(5736): 887-888.

[5] Roman GT, Chen Y, Viberg P, et al. Single-cell manipulation and analysis using microfluidic devices. Anal Bioanal Chem 2007; 387(1): 9-12.

[6] Pamula V. Digital microfluidics by electrowetting. Duke University; 2007 [cited 2007 Dec 23]. Available from: http://www.ee. duke.edu/research/microfluidics

[7] Cho SK, Moon H, Kim CJ. Creating, transporting, cutting and merging liquid droplets by electrowetting-based actuation for digital microfluidic circuits. J Microelectromech Syst 2003; 12(1): 7080.

[8] Fowler J, Moon H, Kim CJ. Enhancement of mixing by dropletbased microfluidics. MEMS 2002, Las Vegas, NV 2002; pp. 97100 .

[9] de Gennes PG. Wetting: statics and dynamics. Rev Mod Phys 1985; 57(3): 827-863.

[10] Mugele F, Baret JC. Electrowetting: from basics to applications. J Phys Condens Matter 2005; 17(28): R705-R774.

[11] Su F, Ozev S, Chakrabarty K. Concurrent testing of digital microfluidics-based biochips. ACM Trans Design Autom Electron Syst 2006; 11(2): 442-464.

[12] Moon H, Cho SK, Garrell RL, et al. Low voltage electrowettingon-dielectric. J Appl Phys 2002; 92(7): 4080-4087.
[13] Fan SK, Hashi C, Kim CJ. Manipulation of multiple droplets on NxM grid by cross-reference EWOD driving scheme and pressurecontact packaging. MEMS 2003, Kyoto, Japan 2003; pp. 694-697.

[14] Shapiro B, Moon H, Garrell RL, et al. Equilibrium behavior of sessile drops under surface tension, applied external fields, and material variation. J Appl Phys 2003; 93(9): 5794-5811.

[15] Yoon JY, Garrell RL. Preventing biomolecular adsorption in electrowetting-based biofluidic chips. Anal Chem 2003; 75(19): 50975102.

[16] Chatterjee D, Hetayothin B, Wheeler AR, et al. Droplet-based microfluidics with nonaqueous solvents and solutions. Lab Chip 2006; 6(2): 199-206.

[17] Egatz-Gómez A, Melle S, García AA, et al. Discrete magnetic microfluidics. Appl Phys Lett 2006; 89(3): 034106.

[18] Park JH, Derfus AM, Segal E, et al. Local heating of discrete droplets using magnetic porous silicon-based photonic crystals. J Am Chem Soc 2006; 128(24): 7938-7946.

[19] Yoon JY. Microdrop manipulations on superhydrophobic surface for latex immunoagglutination assays using backscattering detection. Paper presented at Institute of Biological Engineering (IBE) 2008 Annual Conference, Chapel Hill, NC, March 6-9, 2008.

[20] Shastry A, Case MJ, Bohringer KF. Directing droplets using microstructured surfaces. Langmuir 2006: 22(14): 6161-6167.

[21] Lucas LJ, Han JH, Chesler J, et al. Latex immunoagglutination for a vasculitis marker in a microfluidic device using static light scattering detection. Biosens Bioelectron 2007; 22(9-10): 2216-2222.

[22] Lucas LJ, Chesler JN, Yoon JY. Lab-on-a-chip immunoassay for multiple antibodies using microsphere light scattering and quantum dot emission. Biosens Bioelectron 2007; 23(5): 675-681.

[23] Han JH, Heinze BC, Yoon JY. Single cell level detection of Escherichia coli in microfluidic device. Biosens Bioelectron 2008; 23(8): 1303-1306.

[24] Srinivasan V, Pamula VK, Fair RB. An integrated digital microfluidic lab-on-a-chip for clinical diagnostics on human physiological fluids. Lab Chip 2004; 4(4): 310-315.

[25] Lindsay S, Vázquez T, Egatz-Gómez A, et al. Discrete microfluidics with electrochemical detection. Analyst 2007; 132(5): 412416.

[26] Lehmann U, Hadjidj S, Parashar VK, et al. Two-dimensional magnetic manipulation of droplets on a chip as a platform for bioanalytical applications. Sens Actuators B 2006; 117(2): 457-463.

[27] Yoon JY. Latex immunoagglutination assay in lab-on-a-chip. Biol Eng 2008; 1(1): 79-94.

[28] Wheeler AR, Moon H, Kim CJ, et al. Electrowetting-based microfluidics for analysis of peptides and proteins by matrix-assisted laser desorption/ionization mass spectrometry. Anal Chem 2004; 76(16): 4833-4838.

[29] Chang YH, Lee GB, Huang FC, et al. Integrated polymerase chain reaction chips utilizing digital microfluidics. Biomed Microdevices 2006; 8(3): 215-225.

[30] Ohashi T, Kuyama H, Hanafusa N, et al. A simple device using magnetic transportation for droplet-based PCR. Biomed Microdevices 2007; 9(5): 695-702.

[31] Pipper J, Inoue M, Ng LFP, et al. Catching bird flu in a droplet. Nat Med 2007; 13(10): 1259-1263.

[32] Beer NR, Hindson BJ, Wheeler EK, et al. On-chip, real-time, single-copy polymerase chain reaction in picoliter droplets. Anal Chem 2007; 79(22): 8471-8475.

[33] Huebner A, Srisa-Art M, Holt D, et al. Quantitative detection of protein expression in single cells using droplet microfluidics. Chem Commun 2007; (12): 1218-1220.

[34] He M, Edgar JS, Jeffries GDM, et al. Selective encapsulation of single cells and subcellular organells into picoliter- and femtolitervolume droplets. Anal Chem 2005; 77(6): 1539-1544. 